TITLE:

\title{
Efficient second harmonic generation in a metamaterial with two resonant modes coupled through two varactor diodes
}

\section{AUTHOR(S):}

Nakanishi, Toshihiro; Tamayama, Yasuhiro; Kitano, Masao

\section{CITATION:}

Nakanishi, Toshihiro ... [et al]. Efficient second harmonic generation in a metamaterial with two resonant modes coupled through two varactor diodes. APPLIED PHYSICS LETTERS 2012, 100(4): 044103.

\section{ISSUE DATE:}

2012-01

URL:

http://hdl.handle.net/2433/160630

\section{RIGHT:}

Copyright 2012 American Institute of Physics. This article may be downloaded for personal use only. Any other use requires prior permission of the author and the American Institute of Physics. The following article appeared in APPLIED PHYSICS 


\section{AD Applied Physics \\ Letters}

\section{Efficient second harmonic generation in a metamaterial with two resonant modes coupled through two varactor diodes}

Toshihiro Nakanishi, Yasuhiro Tamayama, and Masao Kitano

Citation: Appl. Phys. Lett. 100, 044103 (2012); doi: 10.1063/1.3679652

View online: http://dx.doi.org/10.1063/1.3679652

View Table of Contents: http://apl.aip.org/resource/1/APPLAB/v100/i4

Published by the American Institute of Physics.

\section{Related Articles}

Acoustic radiation efficiency of a periodically corrugated rigid piston

Appl. Phys. Lett. 101, 104103 (2012)

Ultrasonic evaluation of early damage of a coating by using second-harmonic generation technique

J. Appl. Phys. 111, 124902 (2012)

Dissipative and hysteresis loops as images of irreversible processes in nonlinear acoustic fields

J. Appl. Phys. 110, 053503 (2011)

Nonlinear vocal fold dynamics resulting from asymmetric fluid loading on a two-mass model of speech Chaos 21, 033113 (2011)

Experimental realization of broadband tunable resonators based on anisotropic metafluids

Appl. Phys. Lett. 98, 244102 (2011)

\section{Additional information on Appl. Phys. Lett.}

Journal Homepage: http://apl.aip.org/

Journal Information: http://apl.aip.org/about/about_the_journal

Top downloads: http://apl.aip.org/features/most_downloaded

Information for Authors: http://apl.aip.org/authors

\section{ADVERTISEMENT}

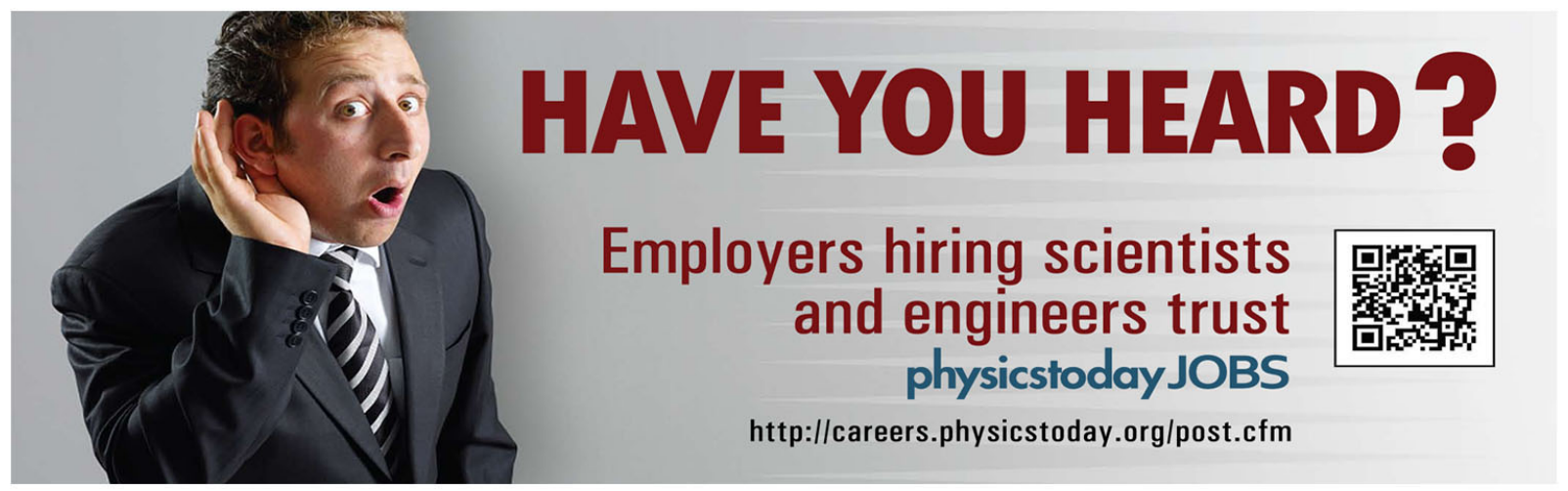




\title{
Efficient second harmonic generation in a metamaterial with two resonant modes coupled through two varactor diodes
}

\author{
Toshihiro Nakanishi, ${ }^{\text {a) }}$ Yasuhiro Tamayama, and Masao Kitano \\ Department of Electronic Science and Engineering, Kyoto University, Kyoto 615-8510, Japan
}

(Received 12 November 2011; accepted 6 January 2012; published online 26 January 2012)

\begin{abstract}
We present an effective method to generate second harmonic $(\mathrm{SH})$ waves using nonlinear metamaterial composed of coupled split ring resonators (CSRRs) with varactor (variable capacitance) diodes. The CSRR structure has two resonant modes: a symmetric mode that resonates at the fundamental frequency and an anti-symmetric mode that resonates at the $\mathrm{SH}$ frequency. Resonant fundamental waves in the symmetric mode generate resonant SH waves in the anti-symmetric mode. The double resonance contributes to effective $\mathrm{SH}$ radiation. In the experiment, we observe $19.6 \mathrm{~dB}$ enhancement in the SH radiation in comparison with the nonlinear metamaterial that resonates only for the fundamental waves. ㄷ 2012 American Institute of Physics. [doi:10.1063/1.3679652]
\end{abstract}

Metamaterials, which are composed of artificial subwavelength structures, exhibit extraordinary electromagnetic properties. Numerous studies have focused on the linear response characteristics of metamaterials. Recent studies have also reported the development of nonlinear media and the control of the nonlinear properties of the metamaterials through the introduction of nonlinear elements into the constituents of metamaterial. High nonlinearity can be achieved in resonant-type metamaterials such as split ring resonators (SRRs) by placing nonlinear elements at locations where the electric (or magnetic) field is concentrated due to the resonance effect. ${ }^{1}$ Nonlinear metamaterials have been studied for property tuning, ${ }^{2-4}$ frequency mixing, ${ }^{5}$ imaging beyond diffraction limit, ${ }^{6}$ and developing bistable media. ${ }^{3,7,8}$ Several kinds of nonlinear metamaterials for generating second harmonic (SH) waves have been reported. Most of the metamaterials are designed such that they resonate with the incident waves or the fundamental waves. ${ }^{2,9-13}$ These are called singly resonant metamaterials. If the structure has resonant modes not only for the fundamental frequency but also for the SH frequency, SH radiation could be enhanced. ${ }^{14,15}$ In this paper, we propose a method to implement metamaterials satisfying the doubly resonant condition, or a doubly resonant metamaterial, using coupled split ring resonators (CSRRs) with two varactor diodes, which generate SH waves due to nonlinearity. There are two resonant modes in the CSRR structure: one for the fundamental waves; the other for the SH waves. They are coupled owing to the nonlinearity of the diodes. The SH oscillation excited in the varactors directly excites the $\mathrm{SH}$ resonance mode through the nonlinearity-assisted coupling. Second harmonic generation through the CSRRs is more efficient than that through the previously proposed metamaterial with two resonant modes, where SH oscillation indirectly excites the resonant mode designed for the SH waves through magnetic coupling. ${ }^{15} \mathrm{We}$ demonstrate experiments in the microwave region to estimate the SHG efficiency of the CSRRs, comparing the singly resonant metamaterial.

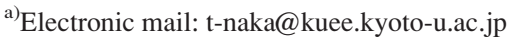

The varactor loaded SRR shown in Fig. 1(a) is a typical example of singly resonant nonlinear metamaterials. It can be modeled as a series resonant circuit composed of an inductor, varactor, and resistor, as shown in Fig. 1(b). The electromotive force induced by the external magnetic field $B$ is represented by the voltage source $V(t)=V_{0} \cos \omega t$ in the circuit model. If we consider the varactor as a linear capacitor, the circuit is a simple harmonic oscillator driven by the external force and the voltage across the capacitor, $v_{C}$, reaches a maximum at the resonant angular frequency $\omega_{0}=1 / \sqrt{L C}$. However, the varactor has nonlinearity in the capacitance and its voltage $v_{C}$ with respect to the charge $q$ can be represented as $v_{C}=q / C(q)=q / C+\alpha q^{2}$. An anharmonic term $\alpha q^{2}$ contributes to the generation of second- or higher-order harmonic waves. Using the perturbation method under a weak nonlinearity condition, ${ }^{16}$ the amplitude of the $\mathrm{SH}$ oscillation is obtained as

$$
\tilde{q}(2 \omega)=\frac{\alpha V_{0}^{2}}{\omega^{3} Z(2 \omega) Z(\omega)^{2}},
$$

where $Z(\omega)=R-\mathrm{i}[\omega L-1 /(\omega C)]$ is the impedance of the circuit. When the circuit resonates at $\omega=\omega_{0},|Z(\omega)|$ takes a minimum value and $|\tilde{q}(2 \omega)|$ is maximized. As a result, the enhanced $\mathrm{SH}$ signal is radiated from the singly resonant

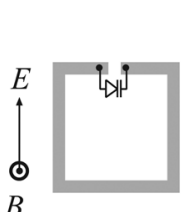

(a)

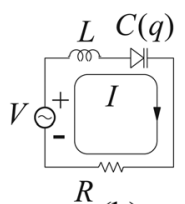

(b)

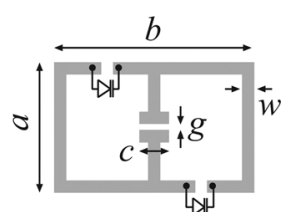

(c)

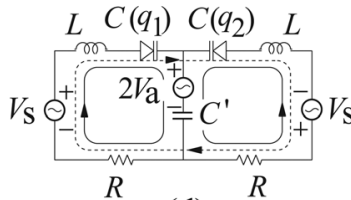

(d)

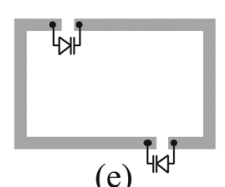

(e)
FIG. 1. (a) Varactor-loaded split ring resonator and (b) its circuit model. (c) Coupled split ring resonator and (d) its circuit model. (e) Split ring resonator as the reference metamaterial. 
metamaterial. Here, we can expect that $|\tilde{q}(2 \omega)|$ is further enhanced by also reducing the impedance at $2 \omega$, i.e., $|Z(2 \omega)|$. This implies that more efficient SHG can be achieved when the metamaterial resonates for both the fundamental and SH frequencies.

For further enhancement of the $\mathrm{SH}$ radiation, we introduce the CSRR shown in Fig. 1(c). Two ring structures share a gap with a capacitance $C^{\prime}$ at the center of the structure. Figure 1(d) represents the circuit model of the CSRR. It should be noted that there are two diodes oppositely directed on the outer ring. We denote the charges of the varactors as $q_{1}$ and $q_{2}$. In terms of the variables, $q_{\mathrm{s}}=q_{1}+q_{2}$ and $q_{\mathrm{a}}=q_{1}-q_{2}$, the equations of motions are expressed as

$$
\begin{gathered}
L \frac{\mathrm{d}^{2} q_{\mathrm{s}}}{\mathrm{d} t^{2}}+R \frac{\mathrm{d} q_{\mathrm{s}}}{\mathrm{d} t}+\frac{q_{\mathrm{s}}}{C}+\alpha q_{\mathrm{s}} q_{\mathrm{a}}=2 V_{\mathrm{s}} \cos \omega t, \\
L \frac{\mathrm{d}^{2} q_{\mathrm{a}}}{\mathrm{d} t^{2}}+R \frac{\mathrm{d} q_{\mathrm{a}}}{\mathrm{d} t}+\frac{q_{\mathrm{a}}}{C_{\mathrm{a}}}+\frac{\alpha}{2} q_{\mathrm{s}}^{2}+\frac{\alpha}{2} q_{\mathrm{a}}^{2}=2 V_{\mathrm{a}} \cos \omega t,
\end{gathered}
$$

where $1 / C_{\mathrm{a}}=1 / C+2 / C^{\prime}$. The excitation voltage $V_{\mathrm{s}}$ is induced by the magnetic flux through the rings and voltage $V_{\mathrm{a}}$ is induced by the electric field at the central gap of the structure. Equations (2) and (3) represent that the symmetric current (dashed line) and anti-symmetric current (solid line) form two resonant modes with resonant angular frequencies of $\omega_{\mathrm{s}}=1 / \sqrt{L C}$ and $\omega_{\mathrm{a}}=1 / \sqrt{L C_{\mathrm{a}}}$, respectively; these modes are coupled through the nonlinearity $\alpha$. We set the gap capacitance $C^{\prime}=2 C / 3$, so that the doubly resonant condition $\omega_{\mathrm{a}}=2 \omega_{\mathrm{s}}$ is satisfied.

We will solve $q_{\mathrm{s}}$ and $q_{\mathrm{a}}$ in the presence of a small nonlinearity $\alpha$, using a perturbative approach. We assume that the solution for $\alpha=0$ is given by $q_{i}^{(0)}=\tilde{q}_{i}^{(0)}(\omega) \mathrm{e}^{-\mathrm{i} \omega t}+$ c.c. $(i=\mathrm{s}, \mathrm{a})$. From Eqs. (2) and (3), the amplitudes of $q_{\mathrm{s}}^{(0)}$ and $q_{\mathrm{a}}^{(0)}$ oscillating at $\omega$ are written as $\tilde{q}_{i}^{(0)}(\omega)=\frac{V_{i}}{\omega Z_{i}(\omega)}(i=\mathrm{s}, \mathrm{a})$, where $Z_{\mathrm{s}}(\omega)=R-\mathrm{i}[\omega L-1 /(\omega C)]$ and $Z_{\mathrm{a}}(\omega)=R-\mathrm{i}$ $\left[\omega L-1 /\left(\omega C_{a}\right)\right]$ are the impedances for the symmetric and anti-symmetric modes, respectively. When the excitation angular frequency $\omega$ is tuned close to $\omega_{\mathrm{s}},\left|\tilde{q}_{\mathrm{s}}^{(0)}\right|$ becomes large due to resonance. On the other hand, the antisymmetric mode is hardly excited; $\tilde{q}_{\mathrm{a}}^{(0)} \sim 0$.

The first-order solution of $q_{\mathrm{a}}$ with respect to $\alpha$ satisfies

$$
L \frac{\mathrm{d}^{2} q_{\mathrm{a}}^{(1)}}{\mathrm{d} t^{2}}+R \frac{\mathrm{d} q_{\mathrm{a}}^{(1)}}{\mathrm{d} t}+\frac{q_{\mathrm{a}}^{(1)}}{C_{\mathrm{a}}}+\frac{\alpha}{2}\left\{q_{\mathrm{s}}^{(0)}\right\}^{2}=0 .
$$

It is found that the resonant oscillation $q_{\mathrm{s}}^{(0)}$ in the symmetric mode induces the $\mathrm{SH}$ current in the anti-symmetric mode through the last term $\frac{\alpha}{2}\left\{q_{\mathrm{s}}^{(0)}\right\}^{2}$. The nonlinearity of the varactor results in a coupling between the two resonant modes. It should be noted that if the diodes are arranged in the same direction on the outer ring, the fundamental current and the induced SH current flow in the symmetric mode, which resonates only for the fundamental wave. From Eq. (4), the amplitude of the SH oscillation is obtained as

$$
\tilde{q}_{\mathrm{a}}(2 \omega)=\frac{\alpha V_{\mathrm{s}}^{2}}{2 \omega^{3} Z_{\mathrm{a}}(2 \omega) Z_{\mathrm{s}}(\omega)^{2}} .
$$

It is easily deduced from Eq. (2) that there are no SH waves in the symmetric mode. This is because the induced $\mathrm{SH}$ electromotive voltages in the two diodes cancel each other due to the anti-symmetric arrangements of the diodes for the symmetric mode. The SH oscillation excited only in the antisymmetric mode forms an electric dipole with magnitude $\tilde{p}=\tilde{q}_{\mathrm{a}}(2 \omega) \bar{g}$, where $\bar{g}$ is an effective dipole length and is determined by the current or charge distributions. The electric dipole oscillation contributes to efficient $\mathrm{SH}$ radiation. Both $\left|Z_{\mathrm{s}}(\omega)\right|$ and $\left|Z_{\mathrm{a}}(2 \omega)\right|$ in Eq. (5) are small, because of the doubly resonant condition, $\omega=\omega_{\mathrm{s}}=\omega_{\mathrm{a}} / 2$. Consequently, the amplitude of the second harmonic oscillation can be significantly enhanced in comparison to the singly resonant metamaterial, where the SH current has to flow through the high impedance $|Z(2 \omega)|$ in Eq. (1).

We fabricated the CSRR illustrated in Fig. 1(c) with $35 \mu$ m-thick copper film on a polyphenylene ether (PPE) substrate with a thickness of $0.8 \mathrm{~mm}$. The dimensions are $a=14 \mathrm{~mm}, b=24 \mathrm{~mm}, c=4 \mathrm{~mm}, g=0.5 \mathrm{~mm}$, and $w=1 \mathrm{~mm}$. For comparison, we also prepared a reference metamaterial, shown in Fig. 1(e), which has the same structure, except for the absence of the central structure and the direction of the diodes. The loop current in this structure also resonates at the same fundamental frequency as that of the CSRR, and the SH electromotive voltages generated in the two varactors contribute to the loop current, which does not resonate. Therefore, the ratio of SHG efficiency between CSRRs and SRRs can be interpreted as the enhancement factor owing to the resonance effect for the $\mathrm{SH}$ waves.

Before SHG demonstration, we conducted transmission measurements to identify the resonant modes and resonant frequency of the metamaterials. We used a waveguide called a stripline TEM-cell, as shown in Fig. $2 .^{16,17}$ The waveguide supports a TEM mode propagating in the $z$ direction. The tapered structures ensure that the wave impedance is maintained at $50 \Omega$ along the waveguide and the tips of the top plate are connected to the input and output ports, which have SubMiniature Type A (SMA) connectors. We placed three SRRs or CSRRs at intervals of $5 \mathrm{~cm}$ inside the waveguide, as shown in Fig. 2. In the transmission spectra, we observed two resonant dips at $1.36 \mathrm{GHz}$ and $2.72 \mathrm{GHz}$ for the CSRRs and a dip at $1.36 \mathrm{GHz}$ for the SRRs. Thus, it is found that the common dips at $1.36 \mathrm{GHz}$ correspond to resonance in the symmetric (or loop-current) mode and the higher resonant mode at $2.72 \mathrm{GHz}$ for the CSRRs is the anti-symmetric mode. This CSRR structure satisfies the doubly resonant condition, $2 \omega_{\mathrm{s}}=\omega_{\mathrm{a}}$.

Figure 3(a) shows the experimental setup to measure the $\mathrm{SH}$ power generated in the metamaterials. A low pass filter (LPF) was used to suppress residual harmonics from the

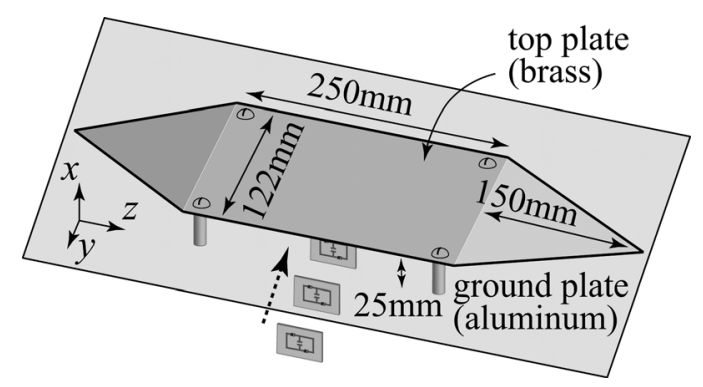

FIG. 2. Schematic of stripline TEM-cell waveguide. 


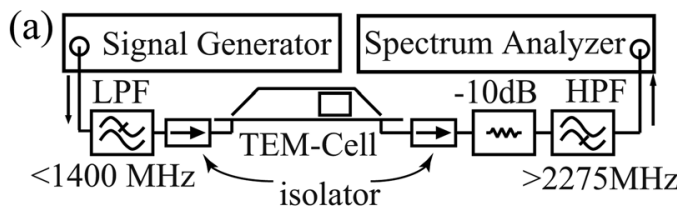

(b)

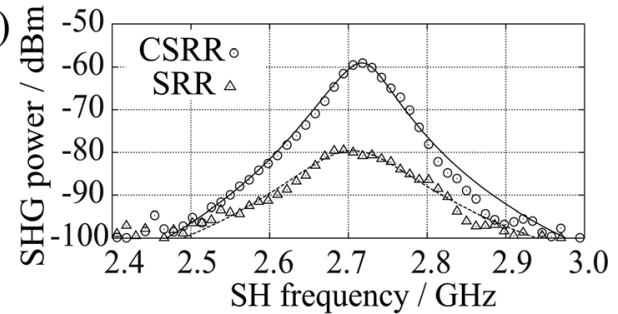

(c)

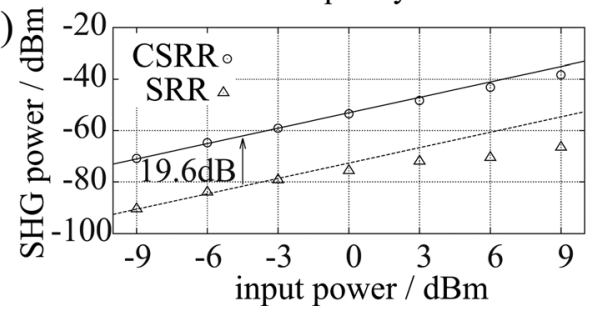

FIG. 3. (a) Experimental setup for SHG measurements. (b) SHG power for $-3 \mathrm{dBm}$ input. (c) $\mathrm{SHG}$ peak power as a function of input power.

signal generator (Agilent N5183A). The output signal containing the $\mathrm{SH}$ wave passes through a high pass filter (HPF) to reject the fundamental wave and is sent to the spectrum analyzer (ADVANTEST U3751). While sweeping the frequency of the input signal, the power density at the SH frequency, or SHG power, was observed. Figure 3(b) shows the SHG power obtained for the CSRR (circles) and SRR (triangles) metamaterials for an input power of $-3 \mathrm{dBm}$. The peaks are found around $2.7 \mathrm{GHz}$ for both cases, and the peak value for the CSRRs is higher than that of the SRRs by about $20 \mathrm{~dB}$. This is clear evidence that SHG in the CSRR metamaterial is significantly enhanced by the resonance at the SH frequency. As shown in Fig. 3(b), we fit experimental data with theoretical curves (a solid line for the CSRR and dashed line for the SRR), which can be derived from the fact that the SHG power is quadratically proportional to Eq. (1) for the SRR and Eq. (5) for the CSRR. The fitting curves reproduce the experimental results well. Figure 3(c) shows the peak values of the SHG spectra. The circles and triangles correspond to the data for the CSRR and SRR, respectively. For weak input power, both SHG spectra exhibit quadratic dependence, which is represented by the solid line for the CSRRs and the dashed line for the SRRs. In this region, the second order perturbation is valid, whereas for greater input power, third-order nonlinearity should be taken into consideration. In fact, in the higher input power region, where the SHG spectra are below the solid or dashed lines, the resonance linewidth broadens and a small resonant frequency shift is observed due to the selfphase modulation. From the above reasons, it is appropriate to estimate the SHG enhancement of the CSRR for weak input power without third- or higher-order nonlinearities. The difference between the solid line and dashed line is $19.6 \mathrm{~dB}$, which corresponds to the enhancement factor owing to the resonance effect for the $\mathrm{SH}$ wave.

As for other types of doubly resonant metamaterials, magnetically coupled split ring resonators have been theoretically analyzed. ${ }^{14}$ Magnetically coupled cut-wire resonators has been studied and $6.6 \mathrm{~dB}$ enhancement of SHG has been achieved. ${ }^{15}$ Both of the metamaterials are composite metamaterial composed of two resonant structures, a primary resonator and secondary resonator, which are magnetically coupled. The SH waves generated in the primary resonator indirectly excite the secondary resonator through magnetic coupling. Roughly speaking, the efficiency of the SHG for these metamaterials in the strong coupling limit could approach that of the CSRR metamaterial, where the SH oscillation directly excites the resonant mode owing to the arrangement of the nonlinear elements. However, it is actually difficult to attain strong magnetic coupling with finite element dimensions and also difficult to optimize the parameters of the metamaterials, considering the resonant frequency shifts induced by strong coupling. The CSRR metamaterial attains a much higher enhancement factor, just by designing the structures to satisfy $\omega_{\mathrm{a}}=2 \omega_{\mathrm{s}}$.

In this paper, we proposed a method to enhance SHG with CSRR metamaterial. The arrangement of the nonlinear elements induces coupling between the two resonant modes, which satisfy the doubly resonant condition. Owing to the direct excitation in the resonant mode for $\mathrm{SH}$ waves, we attained significant enhancement of up to two orders of magnitude. The precise and quantitative evaluation of SHG efficiencies and optimization of the CSRR structure are left as future problems. We expect that metamaterials with nonlinearity-assisted coupling could be employed for investigating other types of nonlinear phenomena.

The present research was supported by Grants-in-Aid for Scientific Research Nos. 22109004 and 22560041, by the Global COE program of Kyoto University and by a research grant from The Murata Science Foundation. One of the authors (Y.T.) would like to acknowledge the support of a Research Fellowship of the Japan Society for the Promotion of Science for Young Scientists.

${ }^{1}$ J. B. Pendry, A. J. Holden, D. J. Robbins, and W. J. Stewart, IEEE Trans. Microwave Theory Tech. 47, 2075 (1999).

${ }^{2}$ I. V. Shadrivov, A. B. Kozyrev, D. W. van der Weide, and Y. S. Kivshar, Appl. Phys. Lett. 93, 161903 (2008).

${ }^{3}$ B. Wang, J. Zhou, T. Koschny, and C. M. Soukoulis, Opt. Express 16, 16058 (2008).

${ }^{4}$ D. A. Powell, I. V. Shadrivov, and Y. S. Kivshar, Appl. Phys. Lett. 95, 084102 (2009).

${ }^{5}$ D. Huang, A. Rose, E. Poutrina, S. Larouche, and D. R. Smith, Appl. Phys. Lett. 98, 204102 (2011).

${ }^{6}$ Z. Wang, Y. Luo, T. Jiang, Z. Wang, J. Huangfu, and L. Ran, Phys. Rev. Lett. 106, 047402 (2011).

${ }^{7}$ R. Yang and I. V. Shadrivov, Appl. Phys. Lett. 97, 231114 (2010).

${ }^{8}$ P.-Y. Chen, M. Farhat, and A. Alù, Phys. Rev. Lett. 106, 105503 (2011).

${ }^{9}$ M. W. Klein, C. Enkrich, M. Wegener, and S. Linden, Science 313, 502 (2006).

${ }^{10}$ M. W. Klein, M. Wegener, N. Feth, and S. Linden, Opt. Express 15, 5238 (2007).

${ }^{11}$ E. Kim, F. Wang, W. Wu, Z. Yu, and Y. Shen, Phys. Rev. B 78, 113102 (2008).

${ }^{12}$ Z. Wang, Y. Luo, L. Peng, J. Huangfu, T. Jiang, D. Wang, H. Chen, and L. Ran, Appl. Phys. Lett. 94, 134102 (2009).

${ }^{13}$ A. Rose, D. Huang, and D. R. Smith, Phys. Rev. Lett. 107, 063902 (2011).

${ }^{14}$ M. V. Gorkunov, I. V. Shadrivov, and Y. S. Kivshar, Appl. Phys. Lett. 88, 071912 (2006).

${ }^{15}$ T. Kanazawa, Y. Tamayama, T. Nakanishi, and M. Kitano, Appl. Phys. Lett. 99, 024101 (2011)

${ }^{16}$ E. Poutrina, D. Huang, and D. R. Smith, New J. Phys. 12, 093010 (2010).

${ }^{17}$ R. E. Collin, Field Theory of Guided Waves (IEEE, New York, 1991). 